\title{
Estudos filogenéticos, taxonômicos e biogeográficos em Chamaecrista, Leguminosae
}

\author{
Phylogenetic, taxonomic and biogeografic studies in \\ Chamaecrista, Leguminosae
}

\begin{abstract}
Tese apresentada ao Instituto de Biociências da Universidade de São Paulo, para a obtenção de Título de Doutor em Ciências, na Área de Botânica.
\end{abstract}

Orientador: Prof. Dr. José Rubens Pirani 
Rando, Juliana Gastaldello

Estudos filogenéticos, taxonômicos e biogeográficos em Chamaecrista, Leguminosae

185 páginas

Tese (Doutorado) - Instituto de Biociências da Universidade de São Paulo. Departamento de Botânica.

1. Filogenia de Leguminosas 2. Campos Rupestres 3. Taxonomia 4. Biogeografia. Universidade de São Paulo. Instituto de Biociências. Departamento de Botânica.

\section{Comissão Julgadora}

Prof.(a) Dr. (a)

Prof.(a) Dr. (a)

Prof.(a) Dr. (a)

Prof.(a) Dr. (a)

Prof. Dr. José Rubens Pirani

Orientador 
Aos meus pais, Renata e Walter a ao meu principal companheiro durante a construção dessa tese, Anselmo. 


\section{AGRADECIMENTOS}

A realização desta tese tem grande importância na minha vida. Considero concluir aqui um ciclo de 12 anos desde o meu primeiro contato com a Botânica Sistemática, momento em qual fui encantada por essa ciência linda que trouxe muitas expectativas e sonhos de seguir a pós-graduação e um dia me tornar uma pesquisadora e professora de Botânica. Nossas vidas são muitas vezes surpreendentes, mudanças inesperadas ocorrem, mudando muitas vezes nossos sentimentos, desejos e criando algumas incertezas. De todas as incertezas e/ou dúvidas que surgiram durante esses 12 anos, algo que eu nunca tive dúvida ou questionei foi o caminho profissional de que optei seguir. A Botânica foi minha maior motivação, uma das coisas mais importantes na minha vida durante todo esse tempo. Com toda essa importância não tenho como não dizer que amo a pesquisa, amo a Botânica e que não me imagino fazendo outra coisa que não seja fazer o que eu faço. Porém, além de toda minha vontade e determinação não poderia nunca ter encerrado esse ciclo sem o apoio dos meus pais, sem aprender com os professores e diversos pesquisadores que conheci durante esse período, e sem a amizade e ajuda de muitos amigos. Agradeço imensamente a tudo e a todos que me ajudaram nessa caminhada, mais especificadamente agradeço:

Ao Instituto de Biociências pelas estruturas oferecida, ao Departamento de Botânica, a todos os professores do Departamento e à curadoria do herbário SPF. Agradeço em especial os funcionários Abel muito querido, Viviane Jono e Roberta Figueiredo. Agradeço também ao Laboratório de Sistemática Molecular da Universidade Estadual de Feira de Santana (Bahia) onde foi realizada a parte molecular do projeto e a todos os funcionários dessa instituição, principalmente ao Ricardo Vilas-Bôas (excelente técnico do laboratório de molecular) e à Téo Nunes (excelente funcionária do herbário HUEFS).

À FAPESP (Fundação de Amparo à Pesquisa do Estado de São Paulo), pela bolsa concedida durante a realização de todo trabalho, tanto pela bolsa nacional e pela reserva técnica (processo 2009/16434-4) quanto à bolsa no exterior BEPE (processo 2013/09374-0) utilizada durante visita do herbários na Europa e ao estágio em Kew Gardens (Inglaterra). 
À CAPES (AUXPE-PROEX 640/2011) pelo apoio em diversas viagens de coleta e compra de alguns materiais de consumo para a etapa molecular.

Ao SISBIOTA (CNPq 563084/2010-3 e FAPESB PES0053/ 2011) por financiar maior parte da etapa molecular realizada em Feira de Santana, Bahia.

Gostaria de dizer um especial obrigado ao orientador do presente trabalho, Prof. José Rubens Pirani, pela disposição e esforço em abrir portas para a execução desse projeto, pelo grande empenho em corrigir projetos, escrever inúmeras cartas de recomendação, corrigir relatórios, submeter artigos, e pelas inúmeras idas e vindas de manuscritos. Sou muito grata de ter tido um orientador em que os olhos brilharam junto com os meus a cada descoberta, a cada discussão sobre as Chamaecrista, por ter contribuído imensamente com o trabalho e com minha formação. Foi um grande privilégio ser sua orientada durante esses anos e sem dúvida sentirei muita falta da sua orientação.

Ao Prof. Luciano Paganucci de Queiroz pela proposta do desafio de trabalhar com Biogeografia de Chamaecrista, pela confiança e por todo apoio cedido à este presente trabalho que ainda renderá muito frutos.

Ao Prof. Vinicius Castro Souza por ter me introduzido na botânica, por ter me inspirado e me dar tantas oportunidades de aprendê-la.

Agradeço ao ICMBio e ao IEF de Minas Gerais pelas licenças de coletas cedidas.

Agradeço à atenção e/ou acolhimento a todos os curadores dos herbários, dos quais necessitei de empréstimos ou que visitei pessoalmente. Em especial aos pesquisadores do New York Botanical Garden que me receberam tão carinhosamente, principalmente, Dr. Wayt Thomas, Dra. Jacquelyn Kallunki e Dr. Douglas Daly. Agradeço imensamente a toda atenção despendida no herbário de Kew, em especial agradeço ao Dr. Gwilyn P. Lewis por ter sido meu supervisor durante $\mathrm{o}$ estágio me auxiliando, discutindo taxonomia e nomenclatura. À Dra. Andreia Flores pelo apoio cedido nas coletas realizadas em Roraima. Ao Dr. Marcelo F. Simon e ao Henrique Moreira por me auxiliaram em uma coleta de campo no Distrito Federal.

Muito obrigada aos companheiros do Departamento de botânica do IBUSP: Augusto G. Oliveira, Beatriz Machado, Benoit Loeville, Caetano Oliveira, Carol Andrino, Carol Siniscalchi, Carol Agostini, Euder G. Martins, Guilherne Antar, Gustavo Heiden, Jennifer Lopes, Juliana de Paula Souza, Juliana Hanna ElOttra, Juliana Lovo, Marcelo Devecchi, Marcelo Pace, Maria Anna Farinaccio, 
Maria Cláudia Medeiros, Matheus Santos, meu querido amigo Maurício Watanabe, Paulo Baleeiro, Paulo Gonella, Suzana Alcântara. Em especial ao Matheus M. Cota e ao Leonardo M. Borges (Caqui) por ter coletado diversas espécies de Chamaecrista que foram incluídas na parte molecular do trabalho, ao Caqui também e pela ajuda na coleta em Brasília. À Maria Fernanda Calió a à Annelise Frazão que me deram uma ajuda imensurável no final da tese com as análises de datação e biogeografia a ao Luiz Henrique Martins Fonseca que leu parte da tese fazendo críticas e sugestões. À Cintia Luiza da Silvia por me ajudar muito na etapa final da tese, com leituras, correções dos textos. Ao Alexandre Zuntini que me ajudou muito com as análises também, dúvidas e ajudas via skype sempre bem disposto a ajudar, valeu He-man!

As meus queridos amigos de Feira de Santana, que foram uma das melhores descobertas durante o doutorado, conhecer tanta gente linda de tantos lugares distintos concentrados em um só lugar, meu lindo amigo Danilo Lima Souza que me ajudou muito no laboratório e depois com a leitura cuidadosa no material examinado do Capítulo 2. À María Cristina Roberts que me deu uma ajuda enorme nas etapas iniciais de laboratório e à Ariane Barbosa que me ajudou o tempo todo desde o treinamento no laboratório até em algumas etapas das análises filogenéticas. Agradece também à Cristiane Snak, Elvia Souza e as muito queridas Cássia Bitencourt, Evelyne Marreira, Gabriela Barros, Marla (Marlíssima) e à baiana mais linda Pétala Ribeiro, a todas pela melhor companhia, risadas e carinho :)

A todos os meus amigos de algum tempo, sempre me apoiando nessa trajetória: minha prima Tati, amiga das antigas Luiza, amigas e amigos esalqueanos Lions, Marina, Patuá, Painço, Pinus, à toda família Basso (Thalão, Thiago e Thaís). Agradeço também aos meus amigos da casa de caridade Luz de Aruanda, uma linda descoberta durante o doutorado que sem dúvida contribuiu muito com a realização da tese, trazendo mais harmonia na minha vida.

Ao Anselmo que durante esses 4 anos foi um grande companheiro, me animando, me ajudando a coletar nos lugares mais inóspitos, me ajudando aprender e entender análises, mostrando um outro lado da biologia e trazendo muita paz, carinho e amor. 
Finalmente, muito obrigada aos meus queridos pais, pois sem o amor, carinho, dedicação e principalmente a confiança jamais teria chegado aqui... em especial agradeço muito à você mãe que tomou frente de cuidar do pai durante o doutorado para que eu pudesse me dedicar plenamente ao doutorado. Vocês são as pessoas mais importantes da minha vida.

"Viva ao povo brasileiro, viva nós!" - João Ubaldo Ribeiro 
Para ser grande

Para ser grande, sê inteiro:

nada teu exagera ou exclui.

Sê todo em cada coisa. Põe quanto és no mínimo que fazes.

Assim em cada lago a lua toda brilha, porque alta vive.

\section{Fernando Pessoa}




\section{SUMÁRIO}

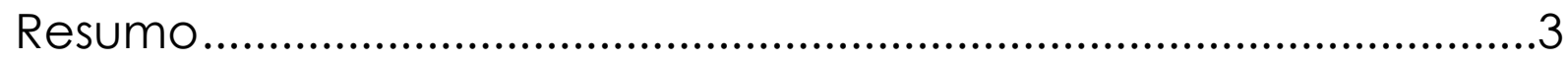

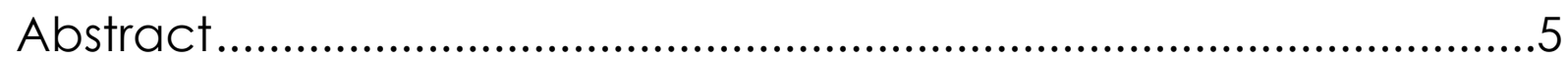

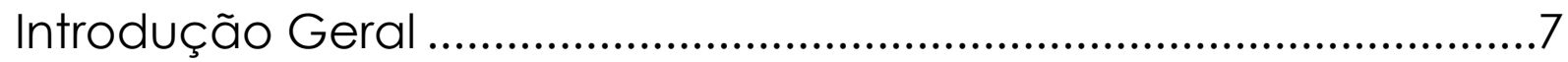

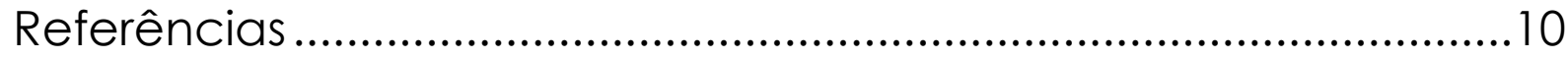

Capítulo 1

Phylogeny of Chamaecrista sect. Chamaecrista ser. Coriaceae

(Leguminosae): a diversified group in the Brazilian highlands. .................... 15

Capítulo 2

2.1. New circumscription, morphology and synopsis of Chamaecrista sect.

Chamaecrista ser. Coriaceae (Benth.) H.S. Irwin \& Barneby ........................75

2.2. Taxonomic novelties in Chamaecrista Moench from Brazil ................ 131

Capítulo 3

Biogeography history of Chamaecrista: ancient occupation versus recent

radiation in Brazilian mountains.

Considerações finais e perspectivas ............................................ 183 


\section{RESUMO}

Neste trabalho apresentamos estudos filogenéticos, taxonômicos e biogeográficos em Chamaecrista, um gênero de Leguminosae com centro de diversidade na região Neotropical. Os estudos de sistemática, filogenia e taxonomia, focaram uma série com 22 espécies (C. ser. Coriaceae), sendo a maioria destas ocorrentes em Campos Rupestres. Já o estudo de biogeografia abrange todo o gênero, visando compreender sua história e diversificação. As etapas de laboratório molecular do trabalho foram realizadas na Universidade Estadual de Feira de Santana, Bahia, em colaboração com o Prof. Dr. Luciano Paganucci de Queiroz.

No capítulo 1, reconstruimos a filogenia de C. ser. Coriaceae utilizando quatro marcadores moleculares e também dados morfológicos. Nossos principais objetivos foram testar a monofilia da série e verificar possíveis padrões de evolução morfológica no grupo. Em nenhuma análise realizada - grupo foi reconstruído como monofilético, porque três espécies apareceram fora do clado que contém os demais representantes da série. Isso levou a uma definição de C. ser. Coriaceae strictu sensu, fortemente sustentada como monofilética nas análises combinadas, sendo que além de sinapomorfias moleculares, duas sinapomorfias morfológicas foram evidenciadas.

No capítulo 2, propomos uma nova circunscrição de C. ser. Coriaceae s.s., baseada nos resultados do capítulo 1, destacando-se a exclusão das três espécies que emergiram distantes deste clado. Apresentamos um estudo morfológico mais detalhado das inflorescências, da morfologia floral e dos nectários extraflorais nessa série, comparativamente aos padrões observados nos demais clados do gênero, e visando a uma reavaliação da circunscrição taxonômica vigente. Compilamos uma sinopse taxonômica da série, incluindo as novidades nomenclaturais, chave de identificação e ilustrações diagnósticas. Chamaecrista ser. Coriaceae s.s. inclui 19 espécies, 2 variedades; três neotipificações são propostas, além de uma nova combinação, dois novos sinônimos. A série é restrita ao Brasil, tendo a maioria 
dos táxons uma distribuição restrita ou endêmica, sobretudo nos Campos Rupestres das serras do Planalto Central. Este capítulo inclui também um artigo publicado durante o doutorado sobre novidades taxonômicas da série, no qual sinonimizamos uma variedade e elevamos três variedades à categoria de espécie.

No capítulo 3, realizamos a datação dos eventos cladogenéticos de Chamaecrista, seguida de análise biogeográfica e evolutiva. Esse estudo abrange mais de um terço das espécies de Chamaecrista. Visamos compreender como e quando se deu a origem e distribuição das espécies do gênero e verificar se esses processos teriam relação com transições morfológicas no grupo. As análises indicam uma origem do grupo relativamente antiga, no Terciário inferior na América do Sul, porém a maioria das espécies parece ter se diversificado mais recentemente e por meio de diferentes processos, sendo a dispersão o mais comum. Como observado em outros grupos de plantas lenhosas neotropicais, Chamaecrista também parece ter origem florestal com uma posterior invasão de áreas abertas, formações onde atualmente está concentrada sua maior diversidade. Alguns caracteres morfológicos derivados no grupo parecem estar relacionados com a ocupação dos habitats savânicos e rupestres, principalmente o surgimento de tricomas glandulares e a transição de hábito. 


\section{Abstract}

This thesis focused on the phylogenetic, taxonomic and biogeographic studies in Chamaecrista, a genus of Leguminosae with the center of diversity in the Neotropics. The studies of systematic, phylogeny and taxonomy, were carried out on 22 species (Chamaecrista series Coriaceae), with most species occurring in Campos Rupestres (Chapter 1 and 2). The study of biogeography covers the entire genus, contributing to the understanding of its history and diversification (Chapter 3). Molecular work were conducted at the Universidade Estadual de Feira de Santana, Bahia, in collaboration with the Prof. Dr. Luciano Paganucci de Queiroz.

In Chapter 1, we reconstructed the phylogeny of $C$. ser. Coriaceae using four molecular markers as well as morphological data. Our objectives were to test the monophyly of the series and to identify possible patterns of morphological evolution in this group. In all analysis the group was not reconstructed as monophyletic because three species appeared outside the clade containing the remaining representatives of the series. This led to a definition of $C$. ser. Coriaceae sensu strictu, strongly supported as monophyletic by several molecular and two morphological synapomorphies in the combined analyzis.

In Chapter 2, we proposed a new circumscription of C. ser. Coriaceae s.s., based on the results of Chapter 1, highlighting the exclusion of the three species emerged out this clade. We present a detailed morphological study of inflorescences, floral structure and extrafloral nectaries in the series, compared to patterns observed in other clades of Chamaecrista, seeking a reevaluation of the current taxonomic circumscription. We produce a complete taxonomic synopsis of the series, including nomenclatural novelties, key identification and diagnostic illustrations. Chamaecrista ser. Coriaceae s.s. includes 19 species and 2 varieties; three neotypification are proposed, a new combination, and two new synonyms. The series is restricted to Brazil, most taxa have a restricted or endemic distribution, especially in the Campos 
Rupestres of the Brazilian central plateau. This chapter also includes an article published during the $\mathrm{PhD}$ about the taxonomic novelties of the series, in which we synonymized a variety and modified the taxonomic category of three varieties to species.

In chapter 3, we carried out the dating of the cladogenetic events in Chamaecrista, and the subsequent biogeographic and evolutionary analysis. The taxa sampling covered more than a third of species. We aim to understand when the genus emerged and how the group has distributed in the Neotropics, especially in the Cerrado and Campos Rupestres. Finally, we also explored whether these processes were related with morphological transitions. Our analyses indicated a relatively ancient origin of the group, at the lower Tertiary in South America, though most species seems to have diversified more recently and through different processes, being the most common species dispersion. As observed in other groups of woody plants from Neotropics, Chamaecrista also seems to have forest origin with a subsequent occupation of open areas, in which the genus actually had its greatest diversity. Some derived morphological characters seem to be correlated with the occupation of drier habitats (Cerrado and Campos Rupestres), especially the appearance of glandular trichomes and the transition of habit. 


\section{INTRODUÇÃO GERAL}

A Sistemática Filogenética, fundamentada na premissa de que as classificações biológicas devem refletir o conhecimento atual sobre as relações de parentesco entre os táxons, é paradigmática na Biologia Comparada moderna (Hennig 1966, Amorim 2002, APG-III, 2009). Discussões recentes nessa escola envolvem questões de refinamento dos métodos analíticos e nos passos das análises, e no aprimoramento de critérios explícitos para a geração de classificações a partir das filogenias disponíveis. Grandes avanços têm sido alcançados nas duas últimas décadas com o advento de novas técnicas moleculares e de tecnologias computacionais, mas, sobretudo, graças à aplicação de métodos de reconstrução filogenética a partir de todos os tipos de dados (Wiens 2009). Por este motivo, a realização de trabalhos taxonômicos robustos depende cada vez mais da obtenção de filogenias consistentes que, em combinação com os métodos taxonômicos clássicos, permitem a elaboração de classificações aprimoradas. Além das classificações, as filogenias também têm tido ampla e crescente aplicação em estudos biogeográficos (e.g. Winkworth \& Donoghue 2005, Wiens 2009), evolutivos (e.g. Donoghue et al. 2003, Friedman et al. 2004) e de diversificação (Ricklefs 2007).

Neste trabalho, visamos a contribuir para o avanço na compreensão da diversidade da biota neotropical, utilizando como estudo de caso em grupo de Leguminosae com centro de diversidade no Domínio do Cerrado brasileiro, analisado com métodos fundamentados no paradigma da Sistemática Filogenética e suas aplicações modernas.

O Cerrado forma um dos domínios fitogeográficos que mais contribuem na formação das paisagens naturais do Brasil (Ratter et al. 2006, Fiaschi \& Pirani 2009). Apesar de grande parte desse domínio ter sofrido forte antropização, exibe ainda uma riqueza elevada de espécies e de endemismos (Ratter et al. 2006). A família de maior riqueza específica no 
Cerrado é Leguminosae, compreendendo 123 gêneros e cerca de 1.174 espécies no domínio (Lima et al. 2014). Os gêneros mais representativos da família no Cerrada são Mimosa L., com ca. 217 espécies, e Chamaecrista Moench com ca. 207 espécies: ambos somam um terço da família nesse domínio (Dutra \& Morim 2014, Souza \& Bortolluzi 2014). Questões de diversificação, evolução e padrões biogeográficos de Leguminosae no Cerrado têm sido ainda pouco exploradas sob métodos explícitos, com escassos estudos baseados em dados moleculares adicionando temporalidade (Simon et al. 2009, Simon \& Pennington 2012). Grande parte dos táxons endêmicos no domínio Cerrado são restritos às serras ao longo da Cadeia do Espinhaço (nos estados de Bahia e Minas Gerais) e na Chapada dos Veadeiros (em Goiás), sobretudo nas formações conhecidas como "Campos Rupestres" (Prance 1994, Giulietti et al. 1997, Simon \& Proença 2000, Fiaschi \& Pirani 2009, Rando \& Pirani 2011). Pouco ainda se sabe sobre a diversificação dessa flora rica em endemismos, isso porque ainda são escassos estudos os filogenéticos e biogeográficos com grupos de plantas dessa região. O presente estudo de Chamaecrista, gênero rico em espécies no Brasil e sobretudo em habitats abertos como os do Domínio do Cerrado, tem por objetivo principal contribuir para completar essa lacuna.

Chamaecrista, grupo considerado monofilético (Conceição et al. 2009), inclui cerca de 330 espécies, distribuídas, principalmente, na América tropical, com menor diversidade na África, Ásia e Austrália e poucas espécies atingindo áreas temperadas (Irwin \& Barneby 1982, Lewis 2005). No Brasil, ocorrem cerca de 230 espécies, sendo que 207 estão concentradas na região do Planalto Central brasileiro, nos estados de Bahia, Goiás e Minas Gerais (Irwin \& Barneby 1978, 1982, Lewis 1987, Souza \& Bortoluzzi 2014). Segundo diversos autores (Irwin \& Barneby 1982, Lewis 1987, Conceição et al. 2001, Souza \& Bortoluzzi 2014, Rando \& Pirani 2011), os estados da Bahia e de Minas Gerais, com 105 espécies restritas a eles, são os principais centros de diversidade do grupo, sendo o Cerrado e os Campos Rupestres os locais de destaque por apresentarem diversas espécies endêmicas. Como exemplo desse endemismo temos Chamaecrista sect. Chamaecrista ser. Coriaceae 8 
(Benth.) H.S. Irwin \& Barneby. Esta série foi descrita por Bentham (1871) e recircunscrita por Irwin \& Barneby (1982), os últimos autores reconheceram 28 táxons (20 espécies e 8 variedades). A série pode ser caracterizada pelo hábito subarbustivo a arbustivo com xilopódio que permite a rebrota de novos ramos depois do fogo, além da presença de nectário extrafloral (Irwin \& Barneby, 1982). Segundo esses autores a série tem a maioria das espécies concentrada no domínio do Cerrado, com uma única espécie restrita à região do Caribe: C. caribaea (Northr.) Britton. No domínio do Cerrado, as espécies de Chamaecrista ser. Coriaceae encontram-se predominantemente nos Campos Rupestres, sendo diversas delas consideradas raras (Giulietti et al. 2009). Apesar da revisão relativamente recente de Chamaecrista (Irwin \& Barneby 1982), persistem questões nomenclaturais a serem resolvidas, além de problemas de circunscrição de algumas variedades que podem ser melhor avaliados com um maior número de informações (e.g. observação das populações em ambiente natural e amplo estudo de coleções atualmente disponíveis).

Diversos trabalhos citam alguns grupos de angiospermas como bons candidatos em estudos que poderão trazer informações sobre a diversificação e história biogeográfica do cerrado e dos Campos Rupestres, e entre eles está Chamaecrista (Fiaschi \& Pirani 2009, Queiroz et al. 2009). Dentro deste contexto apresentamos nesta tese estudos filogenéticos, taxonômicos e biogeográficos neste gênero. Os estudos de filogenia e taxonomia estão focados nas espécies de Chamaecrista ser. Coriaceae, que ocorrem predominantemente nos Campos Rupestres, e os estudos biogeográficos abrangem todo o gênero. 


\section{Referências}

AMORIM, D.S. 2002. Fundamentos de Sistemática Filogenética. Holos, Ribeirão Preto, São Paulo.

ANGIOSPERM PHYLOGENYhylogeny Group (2009). An update of the Angiosperm Phylogeny Group classification for the orders and families of flowering plants: APG III. Botanical Journal of the Linnean Society 161: 105-121.

CONCEIÇÃO, A.S.; QUEIROZ, L.P. \& LEWIS, G.P. 2001. Novas espécies de Chamaecrista Moench (Leguminosae - Caesalpinioideae) da Chapada Diamantina, Bahia, Brasil. Sitientibus série Ciências Biológicas 1: 112-119.

DONOGHUE, M.J.; BELL, C.D. \& WINKWORTH, R.C. 2003. The evolution of reproductive characters in Dipsacales. International Journal of Plant Sciences 164: S453-S464.

DUTRA, V.F. \& MORIM, M.P. 2014. Mimosa in Lista de Espécies da Flora do Brasil. Jardim Botânico do Rio de Janeiro. Disponível em: <http://floradobrasil.jbrj.gov.br/jabot/floradobrasil/FB23084>. Acesso em: 20 Abr. 2014.

FIASCHI, P. \& PIRANI, J.R. 2009. Review of plant biogeographic studies in Brazil. Journal of Systematics and Evolution 47: 477-496

FRIEDMAN, W.E.; MOORE, R.C. \& PURUGGANAN, M.D. 2004. The evolution of plant development. American Journal of Botany 91: 1726-1741.

GIULIETTI, A.M.; PIRANI, J.R. \& HARLEY, R.M. 1997. Espinhaço Range Region, Eastern Brazil. In Davis S.D., Heywood, V.H., Herrera-McBryde, O., Villa-Lobos, J. \& Hamilton, A.C. (eds) Centres of Plant Diversity: A guide and strategy for their conservation. Vol. 3. p. 397-404.

GIULIETTI, A.M.; RAPINI, A.; ANDRADE, M.J.G.; QUEIROZ, L.P. \& SILVA, J.M.C. 2009. Plantas raras do Brasil. Belo Horizonte, Conservação Internacional; Feira de Santana, UEFS.

HENNIG, W. 1966. Phylogenetic Systematics. University of Illinois Press, Urbana, Illinois, $263 \mathrm{p}$

IRWIN, H.S. \& BARNEBY, R.C. 1978. Monographic studies in Cassia (Leguminosae Caesalpinioideae), III. Sections Absus and Grimaldia. Memoir of New York Botanical Garden 30: 1-277. 
IRWIN, H.S. \& BARNEBY, R.C. 1982. The American Cassinae: a synoptical revision of Leguminosae, Tribe Cassieae subtribe Cassinae in the New World. Memoirs of New York Botanical Garden 35: 636-918.

LEWIS, G.P. 1987. Legumes of Bahia. Royal Botanic Gardens Kew. Great Britain.

LEWIS, G.P. 2005. Tribe Cassieae. In Lewis, G.P.; Schrire, B.; Mackinder, B.; Lock, M. (eds.) Legumes of the World. Royal Botanic Gardens, Kew.

LIMA, H.C. de; QUEIROZ, L.P.; MORIM, M.P.; SOUZA, V.C.; DUTRA, V.F.; BORTULOZZI, R.L.C.; IGANCI, J.R.V.; FORTUNATO, R.H.; VAZ, A.M.S.F.; SOUZA, E.R. de; FILARDI, F.L.R.; VALLS, J.F.M.; GARCIA, F.C.P.; FERNANDES, J.M.; MARTINS DA SILVA, R.C.V.; PEREZ, A.P.F.; MANSANO, V.F.; MIOTTO, S.T.S.; TOZZI, A.M.G.A.; MEIRELES, J.E.; LIMA, L.C.P. ; OLIVEIRA, M.L.A.A.; FLORES, A.S.; TORKE, B.M.; PINTO, R.B.; LEWIS, G.P.; BARROS, M.J.F.; SCHUTZ, R.; PENNINGTON, T.; KLIGAARD, B.B.; RANDO, J.G.; SCALON, V.R.; CARDOSO, D.B.O.S.; COSTA, L.C. da; SILVA, M.J. da; MOURA, T.M.; BARROS, L.A.V. de; SILVA, M.C.R.; QUEIROZ, R.T.; SARTORI, A.L.B.; CAMARGO, R. A.; LIMA, I.B. 2014. Fabaceae in Lista de Espécies da Flora do Brasil. Jardim Botânico do Rio de Janeiro. Disponível em: <http://floradobrasil.jbrj.gov.br/jabot/floradobrasil/FB1 15>. Acesso em: 20 Abr. 2014.

PENNINGTON, R.T.; RICHARDSON, J.E. \& LAVIN, M. 2006. Insights into the historical construction of species-rich biomes from dated plant phylogenies, neutral ecological theory and phylogenetic community structure. New Phytologist 172: 605-616.

PRANCE, G.T. 1994. A comparison of the efficacy of higher taxa and species numbers in the assessment of biodiversity in the neotropicos. Philosophical Transactions of the Royal Society of London 345: 89-99.

RANDO, J.G. \& PIRANI, J.R. 2011. Padrões de distribuição geográfica das espécies de Chamaecrista sect. Chamaecrista ser. Coriaceae (Benth.) H.S. Irwin \& Barneby (Leguminosae - Caesalpinioideae). Revista Brasileira de Botânica 34: 499-513.

RATTER, J.A., BRIDGEWATER, S. \& RIBEIRO, J.F. 2006. Biodiversity patterns of the woody vegetation of the Brazilian Cerrado. In: Pennington R.T., Lewis G.P. \& Ratter J.A. eds. Neotropical savannas and seasonally dry forests: plant diversity, biogeography and conservation. The Systematics Association Special Volume, Series 69. Boca Raton: CRC Press. 31-66. 
RIBEIRO, L.P.; RAPINI, A.; SILVA, U.C.S.; KONNO, T.U.P.; DAMASCENA, L.D.; VAN DEN BERG, C. 2012. Spatial analyses of the phylogenetic diversity of Minaria (Apocynaceae): assessing priority areas for conservation in the Espinhaço Range, Brazil. Systematics and Biodiversity. 10:317-331.

RICKLEFS, R.E. 2007. Estimating diversification rates from phylogenetic information. Trends in Ecology and Evolution. 22: 601-610.

SIMON, M.F., GRETHER, R., QUEIROZ, L.P., SKEMA, C., PENNINGTON, R.T. \& HUGHES, C.E. 2009. Recent assembly of the Cerrado, a neotropical plant diversity hotspot, by in situ evolution of adaptations to fire. Proceedings of the National Academy of Sciences of the United States of America 106: 1-6.

SIMON, M.F. \& PROENÇA, C. 2000. Phytogeographic patterns of Mimosa (Mimosoideae, Leguminosae) in the Cerrado biome of Brazil: an indicator genus of high-altitude centers of endemism? Biological Conservation 96: 279296.

SIMON, M.F. \& PENNINGTON, R.T. 2012. Evidence for adaptation to fire regimes in the tropical savannas of the Brazilian Cerrado. International Plant Sciences 173: $711-723$.

SOUZA, V.C. \& BORTULLOZI, R.L.C. 2014. Chamaecrista in Lista de Espécies da Flora do Brasil. Jardim Botânico do Rio de Janeiro. Disponível em: <http://floradobrasil.jbrj.gov.br/jabot/floradobrasil/FB22876>. Acesso em: 20 Abr. 2014

QUEIROZ, L.P.; SANTOS, A.K.; CARNEIRO, D.S.; SOUZA, E.R.; CONCEIÇÃO, A.S.; SIMON, M.F. MACHADO, M.S.; ANDRADE, M.J.G.; GIULIETTI, A.M.; RAPINI, A.; VAN DEN BERG, C. \& CONCEIÇÃO, A. 2009. Por que há tantas espécies raras nos Campos Rupestres da Cadeia do Espinhaço? - o que as filogenias podem nos responder. pp. 844-847. Resumo do $60^{\circ}$ Congresso Nacional de Botânica, Feira de Santana, Bahia.

WIENS, J.J. 2009. Paleontology, genomics, and combined-data phylogenetics: can molecular data improve phylogeny estimation for fossil taxa. Systematic Biology 58: 87-99.

WINKWORTH, R.C. \& DONOGHUE, M.J. 2005. Viburnum phylogeny base don combined molecular data: implications for taxonomy and biogeography. American Journal of Botany 92: 653-666. 


\section{CONSIDERAÇÕES FINAIS E PERSPECTIVAS}

A tese de doutorado contém três capítulos, quatro manuscritos, um já publicado e outros três em formato de manuscritos para publicação. A tese está estruturada de modo a manter uma ligação entre os capítulos: Filogenia, Taxonomia e Biogeografia e, de acordo com essa estrutura, temos as seguintes conclusões:

Capítulo 1. Chamaecrista ser. Coriaceae, como atualmente circunscrita, não constitui um clado. Sua nova definição como grupo monofilético, aqui proposta, envolve a exclusão de três espécies: Chamaecrista caribea, C. roraimae e C. venulosa. C. ser. Coriaceae strictu sensu é formada agora por 19 espécies exclusivas do Brasil e restritas aos Campos Rupestres. Tais espécies apresentam como sinapomorfia molecular diversas deleções no DNA plastidial, e como sinapomorfias morfológicas uma disposição espacial peculiar dos estames e a presença de xilopódio. Embora as análises filogenéticas se baseiem em quatro marcadores moleculares e em dados morfológicos, as relações dentro do clado Coriaceae s. s. têm baixa sustentação e pouca resolução, o que pode estar fortemente relacionada a uma recente diversificação do grupo.

Capítulo 2. Neste tratamento taxonômico, propomos a nova circunscrição de Chamaecrista ser. Coriaceae, baseada nos resultados da filogenia apresentada no Capítulo 1. As análises morfológicas revelaram que alguns caracteres antes negligenciados possuem valor taxonômico. A sinopse inclui as informações taxonômicas fundamentais acerca das 19 espécies agora reconhecidas na série, todas exclusivas do Brasil e restritas aos Campos Rupestres. Propomos três neotipificações, dois novos sinônimos e uma nova combinação. Apresentamos também uma chave de identificação das espécies, comentários taxonômicos, nomenclaturais e sobre a distribuição geográfica, assim como ilustrações de caracteres diagnósticos. 
Capítulo 3. O estudo biogeográfico de Chamaecrista baseia-se em uma amostragem de mais de um terço das espécies conhecidas no gênero, utilizando três marcadores moleculares. A datação molecular dos eventos cladogenéticos, aqui realizada por meio de calibração baseada em um registro fóssil, possibilita a geração de hipóteses robustas sobre história biogeográfica do gênero, cuja origem parece remontar ao Terciário Inferior mas com diversificação mais recente. A reconstrução ancestral de áreas e de caracteres no cronograma, efetuada por meio de critérios de máxima verossimilhança, aponta que Chamaecrista provavelmente teve origem em áreas florestais e, posteriormente, conquistou áreas abertas, savânicas, onde teria experimentado sua maior diversificação. Um padrão previamente esperado envolvia hipótese de migração de Chamaecrista diretamente de florestas para o Cerrado e para a Caatinga e posteriormente colonização dos Campos Rupestres, onde está concentrada a maior diversidade do gênero. No entanto, os dados aqui obtidos sugerem que a invasão dos habitats abertos deve ter ocorrido inicialmente ao longo de áreas ligadas à Cadeia do Espinhaço, que efetivamente é um sistema orográfico geologicamente antigo. A partir dos Campos Rupestres, algumas espécies de Chamaecrista teriam expandido para outros ambientes, como o Cerrado e a Caatinga. Essa hipótese é instigante por mostrar uma ocupação antiga dos Campos Rupestres por um táxon cuja diversificação seria bem recente. A ideia é congruente com alguns trabalhos documentando eventos de extinção e substituição das espécies (species turnover) nos Campos Rupestres, mas ainda são necessários mais estudos filogenéticos com abordagens integradas sobre outros grupos de plantas para corroboração.

Como perspectivas futuras, acreditamos que o primeiro e o segundo capítulo estejam bem estruturados necessitando de poucas alterações para serem submetidos para publicação. No entanto, o terceiro capítulo necessita de mais esforços visando a obter algumas sequências faltantes, incrementar as análises e aprofundar a discussão. Por muito tempo as análises de Biogeografia Histórica basearam-se em padrões espaciais (e.g. análise de 184 
parcimônia de Brooks, análises de componentes) ou em eventos (e.g. DIVA Dispersal-vicariance analysis). Entretanto, até mesmo o criador de DIVA, Frederick Ronquist, interpreta como "um grande retrocesso na área acadêmica que pesquisadores ainda usem DIVA". Isso se deve principalmente ao fato de muitos biogeógrafos modernos (e.g. Michael Donoghue) afirmarem que a história biogeográfica sem incorporação de tempo nas análises podem gerar hipóteses pouco consistentes, sendo imprescindível que sejam feitas comparações apenas entre topologias cujos eventos tenham boa evidência de contemporaneidade. Mais recentemente, o grande incremento de dados moleculares disponíveis, o desenvolvimento de análises de verossimilhança e de métodos bayesianos, e a possibilidade de incorporação da temporalidade nas topologias, levaram a grande avanço nas análises biogeográficas, que estão mais refinadas e robustas. Na tese de doutorado testamos três modelos e três time frames, mas isso pode ser melhor detalhado e outras hipóteses podem ser adicionadas ou melhor elaboradas. Atualmente outras ferramentas para análises biogeográficas também já estão disponíveis, como o pacote na linguagem R, o BioGeoBears, que além de todos os parâmetros testados no programa Lagrange (Likelihood Analysis of Geographic Range Evolution) traz a opção de adicionar o efeito fundador nas análises. Enfim, esse terceiro capítulo ainda deverá ser explorado mais amplamente nas análises biogeográficas e de diversificação, e assim provavelmente trará boa contribuição para história biogeográfica da biota neotropical. 